\title{
Performance Evaluation of a Reconfigurable Optical Add Drop Multiplexer Design for High-Order Regular and Offset-QAM Signals
}

\author{
Kyriakos Vlachos $^{1}$, Filipe Ferreira ${ }^{2}$, and Stelios Sygletos $^{2}$ \\ ${ }^{1}$ Computer Engineering and Informatics Dept, University of Patras, GR26500, Rio, Greece \\ ${ }^{2}$ Aston Institute of Photonic Technologies, Aston University, Birmingham, UK \\ e-mail: kvlachos@ceid.upatras.gr
}

\begin{abstract}
In this paper, we investigate the performance of a Reconfigurable Optical Add Drop Multiplexer (ROADM) architecture, that is suitable of supporting high-order regular as well as offset based QAM signals. In the proposed design, a bank of Phase Sensitive Amplification (PSA) subsystems is introduced for processing offset QAM signals, while sub-channel extraction is achieved via waveform splitting and coherent subtraction. Numerical studies have been carried out to validate functional performance, when processing high-order regular and offset based QAM signals.
\end{abstract}

Keywords: add-drop multiplexing, QAM signals, coherent detection, OFDM super-channels.

\section{INTRODUCTION}

ROADMs play a crucial role in demultiplexing, routing and generally processing (adding/dropping) of wavelengths in transparent optical networks [1]. Since their early development, ROADMs have been evolved from plain wavelength blockers to multi-degree wavelength selective switches and are widely deployed to ease monitoring and planning as well as confer better bandwidth utilization and wavelength management to network administrators. Classic ROADMs, particularly those that are using directional Wavelength Selective Switches (WSS), target DWDM transmission systems for typical add/drop operations of the employed wavelength channels. However, with the introduction of orthogonal frequency division multiplexing (OFDM) [2] as well as Nyquist pulse shaped signals [3] current ROADM solutions are inadequate. On one hand, Nyquist-WDM super channels require the implementation of extremely sharp optical filters [4] to enable add-drop multiplexing of signals within their band. On the other hand, OFDM super-channels require the implementation of optoelectronic [5] or all-optical [6] interferometric solutions, due to the spectral overlapping of their sub-channels. Clearly alloptical solutions are much preferred. However, unless there will be a sacrifice in spectral efficiency, through the use of guard-band interval between the OFDM sub-channels, the inter-symbol interference (ISI) induced crosstalk from the sub-channel multiplexing process will limit the scalability of the system to low constellation orders [7]. In [8], it has been shown that by offsetting the in-phase and quadrature tributaries by half the symbol period, the ISI crosstalk can be eliminated even using practical signal spectral profile or pulse shape. This may enhance spectral efficiency, employing high-order offset-QAM, with performance approaching the theoretical limits.

In this paper, we investigate the performance and extend the operation of a recently proposed ROADM architecture, that can support high-order regular- as well as offset-based QAM signals. This is accomplished, by employing a nonlinear phase sensitive selection process, which allows separating the in-phase from the quadrature component of the replicated channel. The architecture is based on linear all-optical methods, such as FFT/i-FFT filtering and time-domain sampling, for creating a replica of the sub-channel signal, a copy of which is detected locally for the dropping process. The node is completed using a simple coherent insertion process, where appropriate frequency locking is based on established carrier extraction techniques.

\section{ROADM Architecture}

The ROADM architecture for regular QAM signals, initially proposed in [7] is depicted in Fig. 1a. The proposed new architecture for processing offset QAM signals, by introducing a bank of Phase Sensitive Amplification (PSA) subsystems, is shown for the first time in Fig. 1b. The PSA sub-system is commissioned to differentially amplify in-phase component (while suppressing the quadrature) of the IQ QAM signal. For both designs, two conventional wavelength selective switches (WSS), one at the input and one at the output of the node, perform the selection of the super-channel and its re-insertion (after its processing) into the output OFDM signals stream, respectively. The WSSs should have a capability for grid-less operation. Within the super-channel to be processed, sub-channel switching is achieved by means of a two-branch modular interferometer structure, denoted as Branch-A and Branch-B (see Fig. 1a and Fig. 1b). The architecture is modular in the sense that additional interferometer sub-paths can be connected in parallel using the available WSS ports to increase the number of super-channels that can be simultaneously processed by the node. 

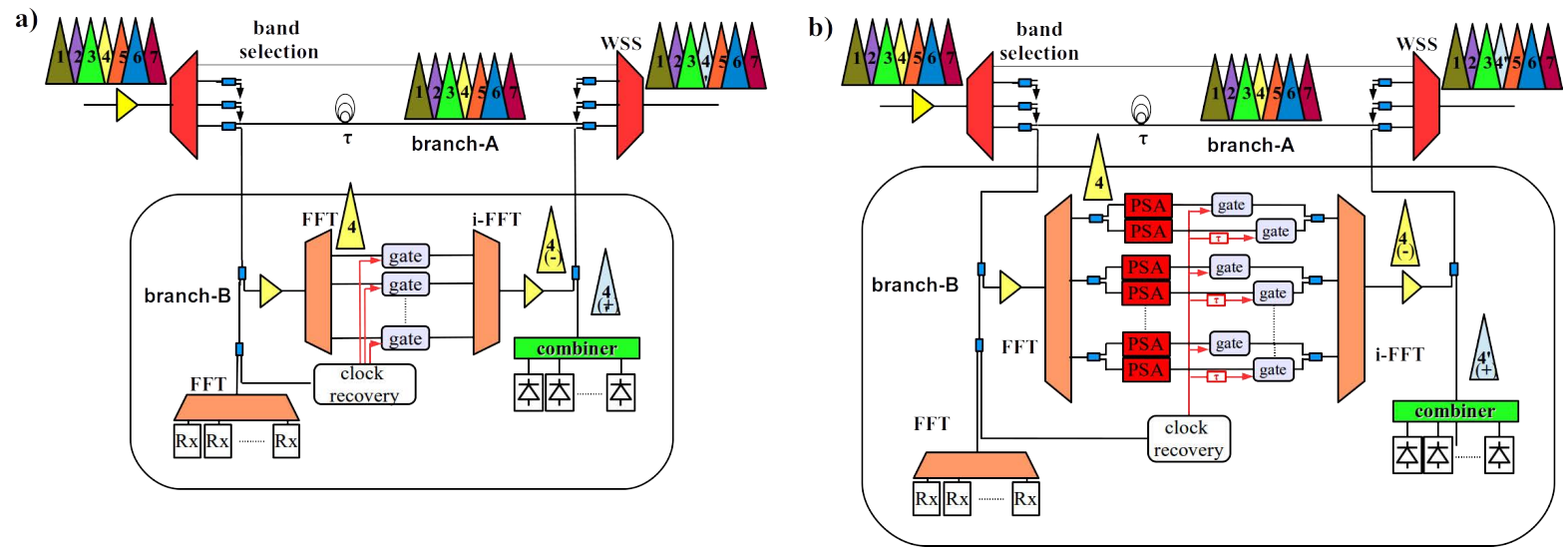

Figure 1: a) ROADM architecture for regular m-QAM signals; b) Extension of the architecture, including Phase Sensitive Amplification subsystems for the quadrature separation, for performing asynchronous sampling on the two quadrature components.

At the node input, the selected super-channel is split into three copies. One copy is dropped to the local receivers and the two remaining copies feed the interferometer branches-A and -B. Sub-channel blocking is facilitated by replicating the corresponding signal waveform in branch-B and interfering it destructively with the super-channel that propagates through branch-A. During the channel replication process, the signals remain in the optical domain, while they are processed in the following three stages. The first stage involves de-multiplexing of the OFDM signal by an optical Fast Fourier Transform (FFT) processor. For the architecture addressing regular m-QAM signals (see Fig. 1a), a subsequent bank of optical gates (one for each sub-channel to be extracted) performs time sampling of the sub-channels that need to be blocked from the through path. The gates are synchronized to a common clock signal, extracted from the super-channel receivers. Each one of them selects a sampling window of minimum crosstalk and feeds the waveform to an optical i-FFT processor, with transfer function $H(f)=\sin c(f T)$, which reshapes the pulses back to their initial symbol duration $T$.

For the case of offset-QAM signals, the architecture is modified by including a set of phase sensitive amplifiers (PSA) for each demultiplexed sub-channel. These are commissioned to perform the separation of the signal's quadrature components. Each PSA has also the capability of suppressing phase distortions that originate from the in-line amplifiers, as well as, Kerr-based nonlinear effects in the transmission link. A bank of optical gates located at the output of each PSA, creates a switching window of finite duration that samples the signal waveform at the areas of minimum crosstalk. In the case of offset QAM signals, the associated switching windows are characterized by a fixed delay difference of $T / 2$. The in-phase and quadrature components of the signal are subsequently recombined and directed to the i-FFT processor, where pulse reconstruction takes place. Subsequently, the recovered waveform is amplified and interfered destructively with the OFDM signal coming from Branch-A. From this process a spectral hole is created leaving an empty position for the new (sub-carrier) channel to be added. All losses, optical delays and relative optical phases should be carefully adjusted to maximize the extinction of the channel extraction. The insertion of the new channels takes place separately, with a bank of laser transmitters aligned to the sub-channel frequencies of the OFDM super-channel.

\section{OFDM CHANNELS PROCESSING CAPABILITIES}

The suitability of the proposed ROADM design for processing (add/drop operation) of OFDM channels has been verified assuming a conventional $10 \mathrm{Gbaud} / \mathrm{s}$ QPSK signal. We have performed extensive numerical simulations to evaluate the performance of the node. For this, we have considered an OFDM super-channel comprising seven optical sub-carriers spaced at $10 \mathrm{GHz}$. The sub-carriers were modulated, according to the simulation scenario under study, either in regular or offset m-QAM format using IQ modulators that were driven by 10 Gbit/s randomly generated binary sequences of rectangular pulses (1's and 0's of equal probability). The electrical signals experienced also a low-pass response from a 1st-order Gaussian filter, giving them a rise-time, which was considered one of the parameters under evaluation. For the case of regular m-QAM signals a frequency Guard-band Interval (GI), expressed as the percentage of the sub-carrier spacing, was also employed between the OFDM sub-channels for increasing the signal robustness against any residual ISI induced crosstalk. For offsetQAM based OFDM signals the use of GI is not applicable

In our study, we have assumed that the optical gate creates rectangular switching windows, synchronized to the de-multiplexed pulse-streams, for which their time duration and contrast ratio could be independently varied. The optical amplifiers within the node had a noise figure equal to $4.5 \mathrm{~dB}$. Signals were detected using an optically pre-amplified coherent receiver with a zero linewidth local oscillator (to allow us to focus on the impact of the node) and an FFT optical demultiplexing filter. Performance was quantified by an iterative Monte-Carlo method with direct error counting. Iterations were performed using each time different data patterns of $2^{15}-1$ bit 
length for the OFDM sub-channels and they were terminated when the BER estimation was statistically expected to have less than $10 \%$ error

Figure 2 depicts the impact on the received OSNR level that corresponds to a BER of $10^{-3}$, as a function of the rise time of the pulses for different constellation orders of $m=\{16,32,64,128\}$ for regular $m$-QAM modulation of each sub-channel in the OFDM super-channel. The results have been taken for different percentages of the guard-band interval $(0 \%$ to $20 \%)$. We can see that as the rise-time increases, significant ISI crosstalk is introduced that degrades heavily the signal performance, especially when scaling to high constellation orders. On the other hand, the introduction of the guard-band interval alleviates the impact of crosstalk and allows migration to high constellation orders, thus allowing the use of commercial available transmitters. For example, a transmitter that can generate optical pulses of $\sim 15 \mathrm{ps}$ rise-time, may enable operation even for 128-QAM modulated sub-channels, when the guard-band interval is $20 \%$.
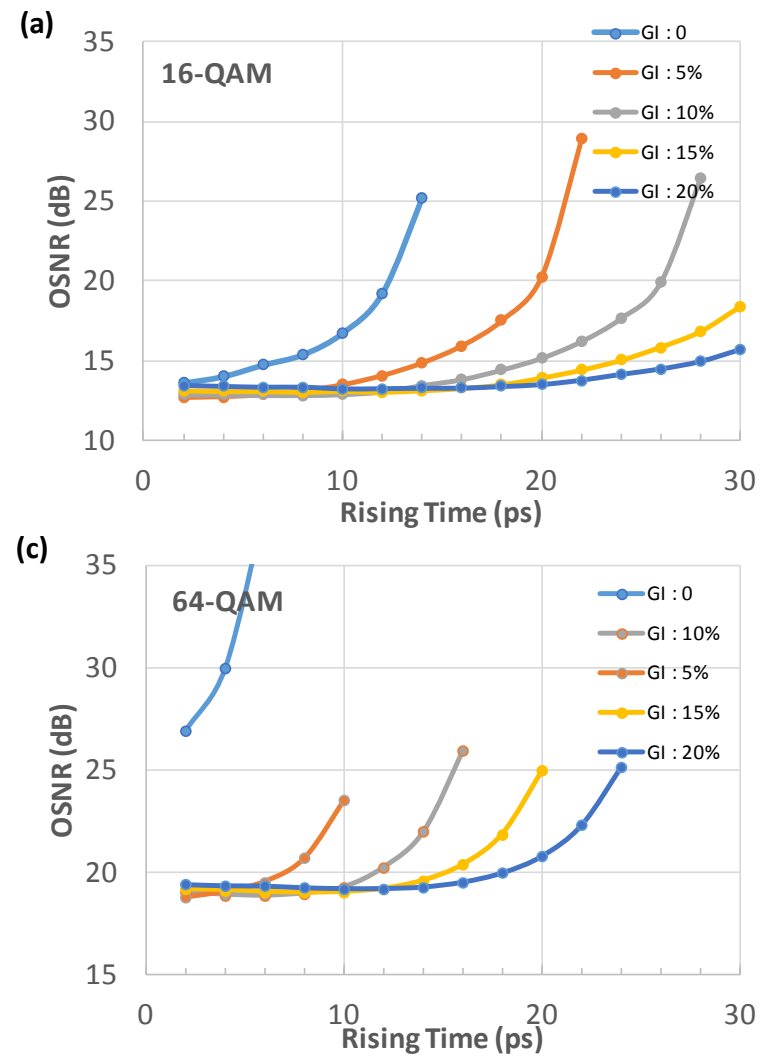
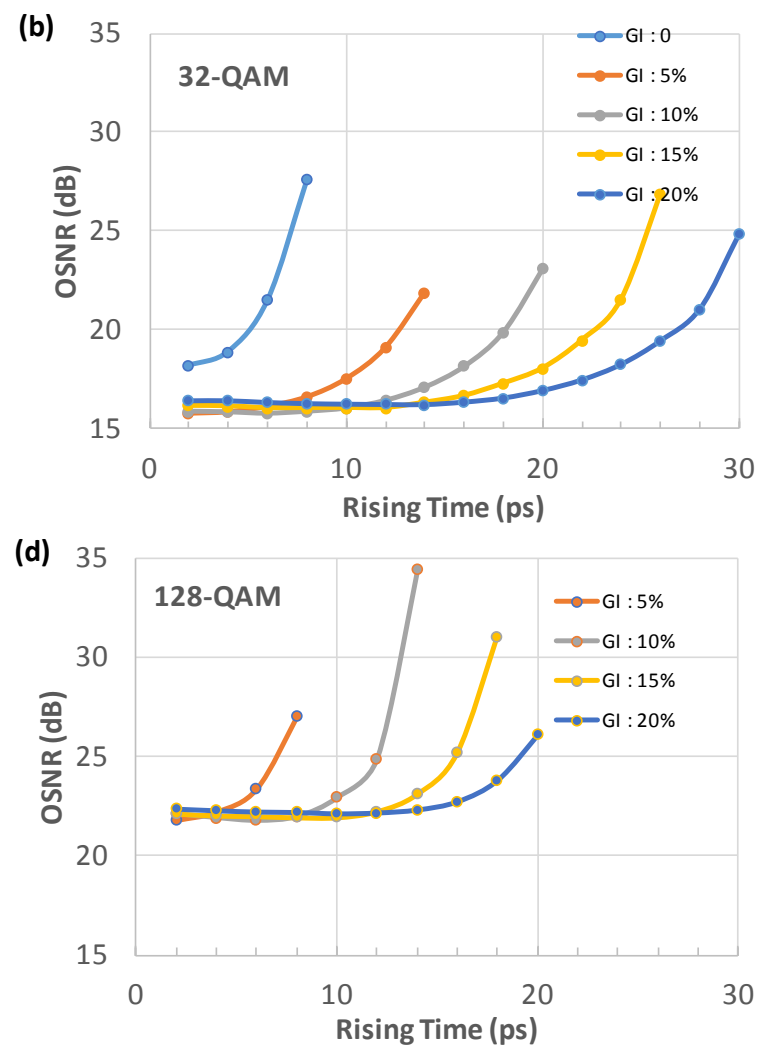

Figure 2. Back-to-back performance of an optical OFDM super channel that consists of seven sub-channels, each carrying regular m-QAM modulation at 10 GBaud. The results depict OSNR levels at the receiver that correspond to a BER of $10^{-3}$, as a function of the pulse rise-time and guard-band intervals (GI), for the constellation orders of a) $m=16, b) m=32$, c) $m=64$ and d) $m=128$.

Next, we analyse back-to-back performance for offset-QAM formats, for the same rise-time values and perform a direct comparison with the regular QAM case. The impact on the received OSNR level for offset $m$ QAM with $m=\{4,16,32\}$ is depicted in Fig. 3a, and for $m=\{64,128\}$ in Fig. 3b. As offset-QAM signals cannot operate with guard-band in OFDM super-channels, direct performance comparison with regular QAM signals is achieved for zero guard-band interval. The results show that using offset-QAMs gives superior performance especially when scaling to large constellation orders. The net gain in OSNR span from $1 \mathrm{~dB}$ to $2 \mathrm{~dB}$ for a pulse rise times below $5 \mathrm{ps}$ and to more than $3 \mathrm{~dB}$ for higher rise times. The gain in performance depends on the modulation order, i.e. the higher order QAMs, the higher the OSNR gain becomes. In particular, for a pulse rise-time of $6 \mathrm{ps}$, the OSNR net gain using an offset rises from $0.1 \mathrm{~dB}$ to $0.9 \mathrm{~dB}$, to $3.1 \mathrm{~dB}, 9.6 \mathrm{~dB}$ and $10 \mathrm{~dB}$ respectively for $m=\{4,16,32,64$ and 128$\}$ QAM. 
(a)

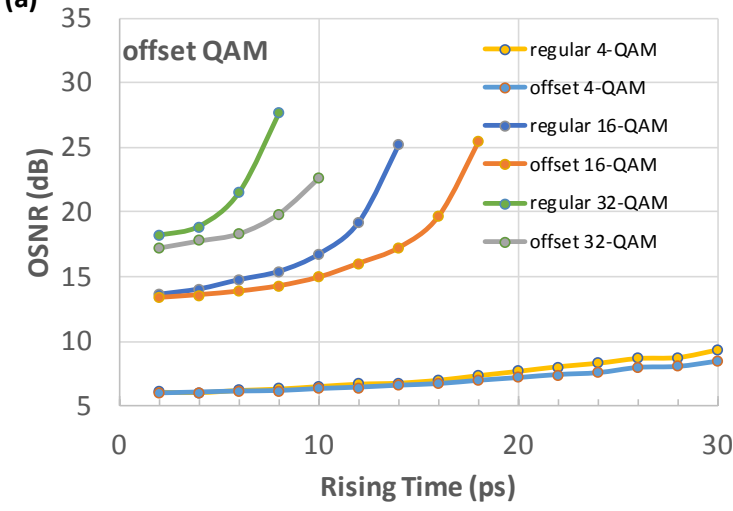

(b)

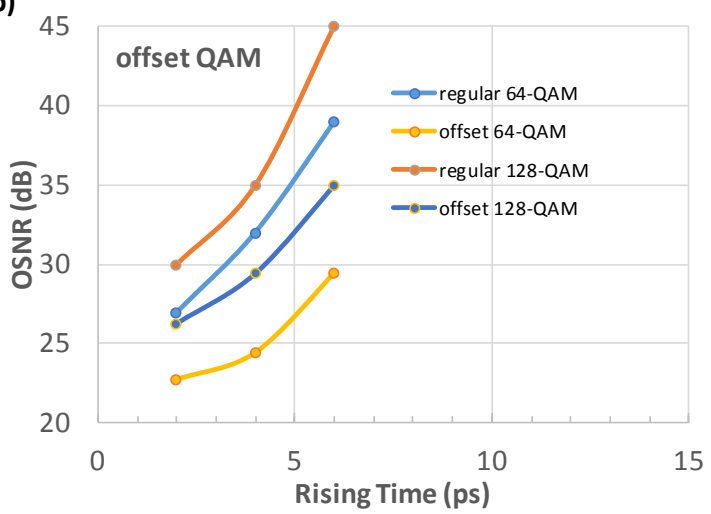

Figure 3. Back-to-back performance of optical OFDM super channel with seven offset m-QAM modulated subchannels at 10GBaud. The results depict OSNR levels at the receiver that correspond to BER of $10^{-3}$, as a function of the pulse rise-time for different constellation order $m$ : (left) $m=\{4,16,32\}$ and (right) $m=\{64,128\}$.

\section{CONCLUSIONS}

In this work, the performance of a ROADM architecture is investigated when processing high-order regular as well as offset-based QAM signals. The proposed design is modular, is based on a interferometer structure and relies on purely all-optical processes, i.e. FFT/i-FFT filtering and time domain sampling. Results have shown the scaling capability of the ROADM scheme for up to offset-32-QAM, whereas for larger constellation orders the performance is heavily limited by the rising time of the transmitter, considering the performance limitations of the commercial available ones.

\section{ACKNOWLEDGEMENTS}

The authors would like to acknowledge Andrew Ellis for useful discussions. The work has been partially supported via EU-ICT project FOX-C (grant number 318415).

\section{REFERENCES}

[1] S. Sygletos, I. Tomkos, and J. Leuthold, "Technological challenges on the road towards transparent networking," J. Opt. Networking, vol. 7, no. 4, pp. 321-350, 2008.

[2] A. D. Ellis and F. C. Gunning, "Spectral density enhancement using coherent WDM," IEEE Photon. Technol. Lett., vol. 15, no. 2, pp. 504-506, 2005.

[3] R. Schmogrow, M. Winter, M. Meyer, D. Hillerkuss, S. Wolf, B. Baeuerle, A. Ludwig, B. Nebendahl, S. BenEzra, J. Meyer, M. Dreschmann, M. Huebner, J. Becker, C. Koos, W. Freude, and J. Leuthold, "Realtime Nyquist pulse generation beyond $100 \mathrm{Gbit} / \mathrm{s}$ and its relation to OFDM," Optics Express, vol. 20, no. 1, pp. 317-337, 2012.

[4] B. Corcoran et al., "Multipass performance of a chip-enhanced WSS for Nyquist-WDM sub-band switching," J. of Lightwave Technology, vol. 34, no. 8, pp. 1824-830, Apr. 2016.

[5] P. J. Winzer, "An opto-electronic interferometer and its use in subcarrier add/drop multiplexing," J. of Lightwave Technology, vol. 31, no. 11, pp. 1775-1782, Jun. 2013.

[6] S. J. Fabbri, S. Sygletos, A. Perentos, E. Pincemin, K. Sudgen, and A. D. Ellis, "Experimental implementation of an all-optical interferometric drop, add and extract multiplexer for superchannels," J. of Lightwave Technology, vol. 33, no. 7, pp. 1351-1357, 2015.

[7] S. Sygletos et al., "Numerical investigation of all-optical add-drop multiplexing for spectrally overlapping OFDM signals," Optics Express, vol. 23, no. 5, pp. 588-5897, 2015.

[8] J. Zhao and A. D. Ellis, "Offset-QAM based coherent WDM for spectral efficiency enhancement," Optics Express, vol. 19, no. 5, pp. 14617, 2011. 